\title{
Molecular and clinical
} characterization of autoimmune polyendocrinopathy-candidiasis-ectodermal dystrophy syndrome (APECED) in Iranian non-Jewish patients: report of two novel AIRE gene pathogenic variants

\author{
Aria Setoodeh ${ }^{1 \dagger}$, Samareh Panjeh-Shahi ${ }^{2+}$, Fariba Bahmani ${ }^{1}$, Fatemeh Vand-Rajabpour ${ }^{3}$, Nazanin Jalilian ${ }^{4}$,
} Fatemeh Sayarifard ${ }^{1}$, Farzaneh Abbasi ${ }^{1}$, Azadeh Sayarifard ${ }^{5}$, Parastoo Rostami ${ }^{1}$, Nima Parvaneh ${ }^{6}$, Haleh Akhavan-Niaki ${ }^{7}$, Mohamadreza Ahmadifard ${ }^{7}$ and Mina Tabrizi ${ }^{3^{*}}$ (1)

\begin{abstract}
Objective: Autoimmune polyendocrinopathy-candidiasis-ectodermal dystrophy syndrome (APECED) is a rare autosomal recessive systemic autoimmune disease caused by mutations in the autoimmune regulator (AIRE) gene. Incidence of this genetic disorder is estimated at 1/90,000-200,000 worldwide and 1/6500-9000 in genetically isolated populations such as Iran. Here, we investigated AIRE gene mutations in eight independent Iranian non-Jewish families.

Methods: We sequenced the coding regions of the AIRE gene and documented mutations which were further confirmed in respective parents.

Results: In total, 11 cases from 8 independent families were recruited. Mucosal candidiasis, Addison's disease and hypoparathyroidism were the most common clinical manifestations in these patients. One novel homozygous splice acceptor mutation (c.308-1G>C), and one novel heterozygous stop-gain mutation (c.1496delC) combined with a known heterozygous c.232T>C missense mutation were found. Moreover, we observed previously described splice donor (c.1095+2T>A), frameshift (c.967-979del), stop-gain (c.415C>T), and missense (c.62C>T) mutations among the patients. All results were co-segregated in parents.

Conclusion: Here, we reported two novel mutations in the AIRE gene leading to APECED. Our data could provide insight into the phenotypic and genotypic spectrum of APECED in the non-Jewish Iranian population. These findings, in addition to future functional assays, can elucidate disease-causing mechanisms related to the AIRE gene and assist in genetic counseling and diagnosis.
\end{abstract}

\footnotetext{
*Correspondence: tabrizi@tums.ac.ir

${ }^{\dagger}$ Aria Setoodeh and Samareh Panjeh-Shahi have contributed equally to this work

${ }^{3}$ Department of Medical Genetics, School of Medicine, Tehran University of Medical Sciences, Tehran, Iran

Full list of author information is available at the end of the article
}

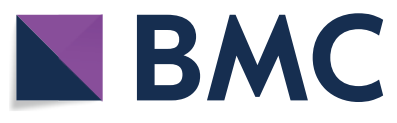

(c) The Author(s) 2022. Open Access This article is licensed under a Creative Commons Attribution 4.0 International License, which permits use, sharing, adaptation, distribution and reproduction in any medium or format, as long as you give appropriate credit to the original author(s) and the source, provide a link to the Creative Commons licence, and indicate if changes were made. The images or other third party material in this article are included in the article's Creative Commons licence, unless indicated otherwise in a credit line to the material. If material is not included in the article's Creative Commons licence and your intended use is not permitted by statutory regulation or exceeds the permitted use, you will need to obtain permission directly from the copyright holder. To view a copy of this licence, visit http://creativecommons.org/licenses/by/4.0/. The Creative Commons Public Domain Dedication waiver (http://creativeco mmons.org/publicdomain/zero/1.0/) applies to the data made available in this article, unless otherwise stated in a credit line to the data. 
Keywords: APECED, APS1, Autoimmunity, AIRE, Addison, Candidiasis

\section{Introduction}

Autoimmune polyendocrinopathy-candidiasis-ectodermal dystrophy syndrome (APECED) [MIM 240300], also known as autoimmune polyglandular syndrome type 1 (APS-1), is a rare autosomal-recessive systemic autoimmune disease often appearing early in life, typically in infants at 3-5 years of age [1]. APECED is known, to date, as an organ-specific human autoimmune disorder affecting several organs and is described to have a Mendelian inheritance pattern. The disease is reported worldwide and occurs in about 1 in 90,000-200,000 people in most populations [2]; however, the lifetime incidence of APECED is exceptionally high in genetically isolated populations such as Iranian Jews [3] with a calculated prevalence of 1/6500-1/9000 based on published documents [4].

This monogenic disorder results from mutations in the autoimmune regulator (AIRE) gene assigned to chromosome 21q22.3 [5]. The genomic structure of the AIRE gene, approximately $13 \mathrm{~kb}$ in length, consists of 14 exons, encoding a polypeptide of 545 amino acids [6]. The gene encodes a transcriptional regulator, constructs nuclear bodies, and interacts with the transcriptional coactivator cAMP-response element-binding protein (CREB). Presence of a conserved nuclear localization signal (NLS) at the $\mathrm{N}$ terminus followed by a SAND domain and four LXXLL motifs are the most substantial characteristics of the AIRE protein [7]. Recent studies have revealed the key role of the encoded protein in shaping central immunological immunity [8]. AIRE is predominantly expressed in the medullary thymic epithelial cells (mTECs); however, to some extent, it is also expressed in rare hematopoietic cells of lymph nodes. AIRE protein provokes expression of a wide array of tissue-restricted proteins in mTECs presented on class I and II major histocompatibility complexes (MHCs) to select non-autoreactive $\mathrm{T}$ cells through thymic media. The "projection of self" process, mediated by mTECs, is critical for deletion of autoreactive $\mathrm{T}$ cells and for development of the main phase in prevention of autoimmunity [9].

Several mutations in the AIRE gene correlate with development of organ-specific autoimmune diseases with a monogenic autosomal recessive inheritance pattern. Among these mutations, the R257X nonsense mutation is common in the Finnish and Eastern European population [10], the Y85C missense mutation has high incidence in isolated populations such as Finns (1:25,000 individuals) [11] and Iranian Jews (1:9000) [12], as well as the R139X nonsense mutation in Sardinian patients
$(1: 14,500)$ [13]. In addition, a 13-bp deletion, located in exon eight (1085-1097), is ubiquitous and can be routinely found in Norwegians, British, and North American populations [14].

Interestingly, signs and symptoms of APECED can widely vary among affected individuals and populations. Presence of primary adrenocortical failure, hypoparathyroidism, and chronic mucocutaneous candidiasis are the most common clinical symptoms for APECED [15]. However, the clinical phenotype can be described as a highly variable combination of autoimmune reactions towards different endocrine and non-endocrine organs [16]. Therefore, researchers believe that differences in clinical manifestations of this pathology might be attributed to the various specific AIRE gene mutations. This hypothesis can emphasize the importance of mutational analysis of the AIRE gene as one of the most important diagnostic tools for early detection of this life-threatening syndrome and the subsequent approach to its treatment [13]. To date, several studies have investigated the frequency of different $A I R E$ gene mutations in Iranian Jews, but there are few studies of AIRE gene mutations among patients with APECED in non-Jewish Iranians [17]. Therefore, the primary aim of this study was to screen the coding regions of the AIRE gene in Iranian patients to determine possible mutations involved in incidence of this syndrome.

\section{Materials and methods Families}

Eight non-Jewish Iranian families, consisting of eleven affected individuals together with their healthy family members, were recruited among patients with APECED admitted to the Children's Medical Center from January to September 2017. In three out of eight families, two affected individuals were studied, and the other five families had only one affected individual in the pedigree. Informed written consent was obtained from all participants and the study was approved by the Institutional Review Board (IRB), with Ethics Committee approval code of IR.MUBABOL.HRI.REC.1397.149.

\section{Diagnostic criteria and clinical data}

The diagnostic criterion was presence of at least two of the following major disease components clearly documented by a physician: hypoparathyroidism (subnormal $\mathrm{Ca}^{2+}$ and supranormal inorganic phosphate concentrations in serum, excluding renal failure), primary adrenocortical failure (primary cortisol and/or aldosterone 
deficiency), and chronic mucocutaneous candidiasis (CMC) [18].

Typical biochemical findings in combination with bone density tests and electrocardiogram (EKG) were used for diagnosis of hypoparathyroidism. Abnormal tooth development was also evaluated in children by an experienced dentist. To confirm adrenal failure, results of the ACTH stimulation tests were interpreted by endocrinologists. To assess the adrenal gland stress response, adrenal response to the adrenocorticotropic hormone was measured. Finally, possible associated hypothyroidism was diagnosed using the thyroid function tests. Liver function was also performed in order to rule out the rare possibility of autoimmune hepatitis. Diagnosis of chronic mucocutaneous candidiasis was made in the occasion of recurrent skin or mucosal candidiasis in absence of diabetes and antibiotic use.

\section{Primer design}

Referenced and designed primer sequences of the AIRE gene (NM_000383) were extracted from the National Center for Biotechnology Information (NCBI) database (Table 1). Then, primers were blasted against the human genome using Primer-BLAST and any possible secondary structures of the designed primers were predicted using Gene Runner software version 3.01.

\section{DNA extraction and PCR amplification}

Genomic DNA was extracted from $5 \mathrm{ml}$ of patient EDTA-treated blood samples using the standard DNA salting out method [19]. Subsequently, all 14 exons of the AIRE gene and their flanking exon-intron

Table 1 Primer sequences used for amplification of exons and exon-intron boundaries of the AIRE gene

\begin{tabular}{lll}
\hline Exon & Forward primer & Reverse primer \\
\hline 1 & CTTTGCTCTTTGCGTGGTCG & GACTATCCCTGGCTCACAG \\
2 & CCTGGGAGCTCCACCCTCTAGT & CACCACTCCGGTTCCAAGTCCA \\
3 & TGGCCAAGGTGTCCAGTTCT & TCTAGTACCCAGAGGAGACC \\
4 & GCAAAGGGACTACCCAGCACT & TAGGACAGGGTCTCAGAGGGCA \\
5 & GCTGCCTGCTTCTGGCATAGA & GGCGTGGTCCTCCTTCCATCTT \\
6 & TCTGCTAGACCCCACCCTG & GCCCCCAGCAGAGCCACT \\
7 & GAACAGCGTTGCCTCTGG & AGTGCCCAGGTAAAGGCA G \\
8 & GGAGTTCAGGTACCCAGAGA & TGACTCAGAACCCCTTTCCA \\
9 & CTGGGGTTTGGGATCTGTC & GGGACATAGTGCTATGGCTGG \\
10 & CCACTCAGTGTGGACGCCTT & TGAATTCATCCGCCCCGTAG \\
11 & GTGAGGCTCCTCACTTGCGCC & TGTGGTTGTGGGTGTATGATGTG \\
12 & TAG & \\
13 & CACACCCCCATACCCCGGA & CTGGTGCAAGCCCTCGAAG \\
14 & AGGTTCTCACCGTCACTCTGT & ACTGACAAGAGGTGGCGCTGT \\
\hline
\end{tabular}

boundaries were investigated in the patients with APECED. In brief, 200 ng of genomic DNA (gDNA) was amplified via a 35-cycle PCR in which the initial 10-min denaturation at $95{ }^{\circ} \mathrm{C}$ was followed by a denaturation at $95{ }^{\circ} \mathrm{C}$ for $30 \mathrm{~s}$, annealing for $30 \mathrm{~s}$, and final extension at $72{ }^{\circ} \mathrm{C}$ for $30 \mathrm{~s}$, in $25 \mu \mathrm{l}$ of total volume containing $10 \times$ Buffer solution, $0.2 \mathrm{~mm}$ of each dNTP, $2.5 \mathrm{~mm}$ $\mathrm{MgCl}_{2}, 0.4 \mu \mathrm{m}$ of each primer, and $2 \mathrm{U}$ of Taq Gold DNA Polymerase (Applied Biosystems, USA). PCR amplifications were performed with specific primers for each exon in a GeneAmp ${ }^{\circledR}$ PCR system 9700 thermal cycler (Applied Biosystems, USA). The PCR products were sequenced by means of a direct chain termination method using BigDye Terminator v1.1 Cycle Sequencing Kits (Applied Biosystems, USA). The sequence products were then purified using the Microcon columns (Millipore) and analyzed on an ABI PRISM 310 automated DNA sequencer (Applied Biosystems, USA).

\section{Validation of the variants and in silico analysis}

Sequence variant numbering was based on the transcript ENST00000291582.6 for AIRE. All novel variants were named according to the guidelines of the Human Genome Variation Society (http://www.hgvs.org/). Upon finding a variant, the following methodology, which is based on the ACMG's guideline for interpretation of sequence variants [20], was adopted in order to validate each variation:

First, segregation analysis was performed for variants in the whole family. In case of novel variants, fifty healthy controls of the same ethnicity were screened along with the patients using Sanger sequencing. Next, an extensive in silico study was performed for the variant.

An extensive search was conducted through databases Ensemble.org, dbSNP (http://www.ncbi.hlm.nih. gov/snp), 1000 genome databases (http://browser.1000g enome.org), HGMD (www.hgmd.cf.ac.uk), Iranome (http://www.iranome.ir), ExAc browser (exac.broadinstitute.org), gnomAD (https://gnomad.broadinstitute. org/), and recently published articles in PubMed.

Possible pathogenic effects of the novel variants were checked by mutation taster (http://www.mutationta ster.org/) and CADD algorithms (https://cadd.gs.washi ngton.edu/snv). In the CADD database, variants with scores more than 14 are categorized as pathogenic.

In addition, BDGP (http://www.fruitfly.org/seq tools/splice.html), Human Splicing finder (http://www. umd.be/HSF3/) and NetGen2 (http://www.cbs.dtu.dk/ services/NetGene2/) were used in order to predict possible effects of splice site mutations. 


\section{Results}

\section{Clinicopathological features}

In total, eleven cases including 7 women and 4 men from 8 unrelated APECED families along with their healthy family members were recruited. The ages ranged from 2 to 26 years and consanguinity was noted in 6 out of the 8 pedigrees. Participant pedigrees and clinical characteristics are depicted in Fig. 1 and Table 2, respectively. The initial and the most frequently observed manifestation of APECED was mucosal candidiasis (CMC) which presented mostly at one year of age, followed by Addison's disease and hypoparathyroidism. On the other hand, vitiligo, chronic malnutrition, autoimmune hepatitis and enamel hypoplasia were the least frequent symptoms detected only once in these families. Detailed information regarding frequency of major symptoms and their distribution among cases are presented in Table 2.

\section{AIRE gene mutations}

In this study, four homozygote and two compound heterozygote pathogenic variants were detected in 6 out of the 8 families studied. These variations included 5 previously reported (c.232T>C, c.967_979delCTGTCCCCT CCGC, c. $1095+2 \mathrm{~T}>\mathrm{A}$, c. $415 \mathrm{C}>\mathrm{T}$ and c.62C $>\mathrm{T}$ ) and two apparently novel variants (c.1496delC and c.308-1G>C). A summary of detected mutations is provided in Table 3 and the location of the mutations at protein level is depicted in Fig. 2. Results of in silico analysis of the splice site variants found in this study are presented in Table 4.
The First novel pathogenic variant, c.1496delC, was observed in IR-APECED-2. In this family, a compound heterozygous mutation, consisting of one novel heterozygous stop-gain mutation (c.1496delC) and a known heterozygous c.232T $>\mathrm{C}$ missense mutation were observed in both affected offsprings. Interestingly, their first cousin parents were heterozygous for c.232T $>C$ (Y78R) and c.1496delC (P499LfsX22) variations, respectively (Fig. 1). The former led to substitution of Trp codon with Arg at codon 78, and the latter deletion would change the amino acid sequence, subsequently causing a premature stop codon. Eventually, both of these heterozygous variations in the gene resulted in malfunctioning of the AIRE protein.

Another novel variant found in our study came from family IR-APECED-4 (Fig. 1). As shown in the pedigree, a homozygous splice acceptor variant (c.308-1G>C) was discovered in the patient (subject II:1) and parents illustrated heterozygous genotypes.

Of note, by sequencing the coding regions of the AIRE gene in patient families Nos. 7 and 8, no mutation was found (families not shown in the pedigree).

\section{Discussion}

Pathogenic variants in the AIRE gene cause APECED, which is described as autoimmune damage to the endocrine glands and multiple ectodermal dystrophies. The chronic or recurrent mucocutaneous candidiasis completes the APECED clinical picture [21]. Studies suggest that the protein encoded by the AIRE gene plays a

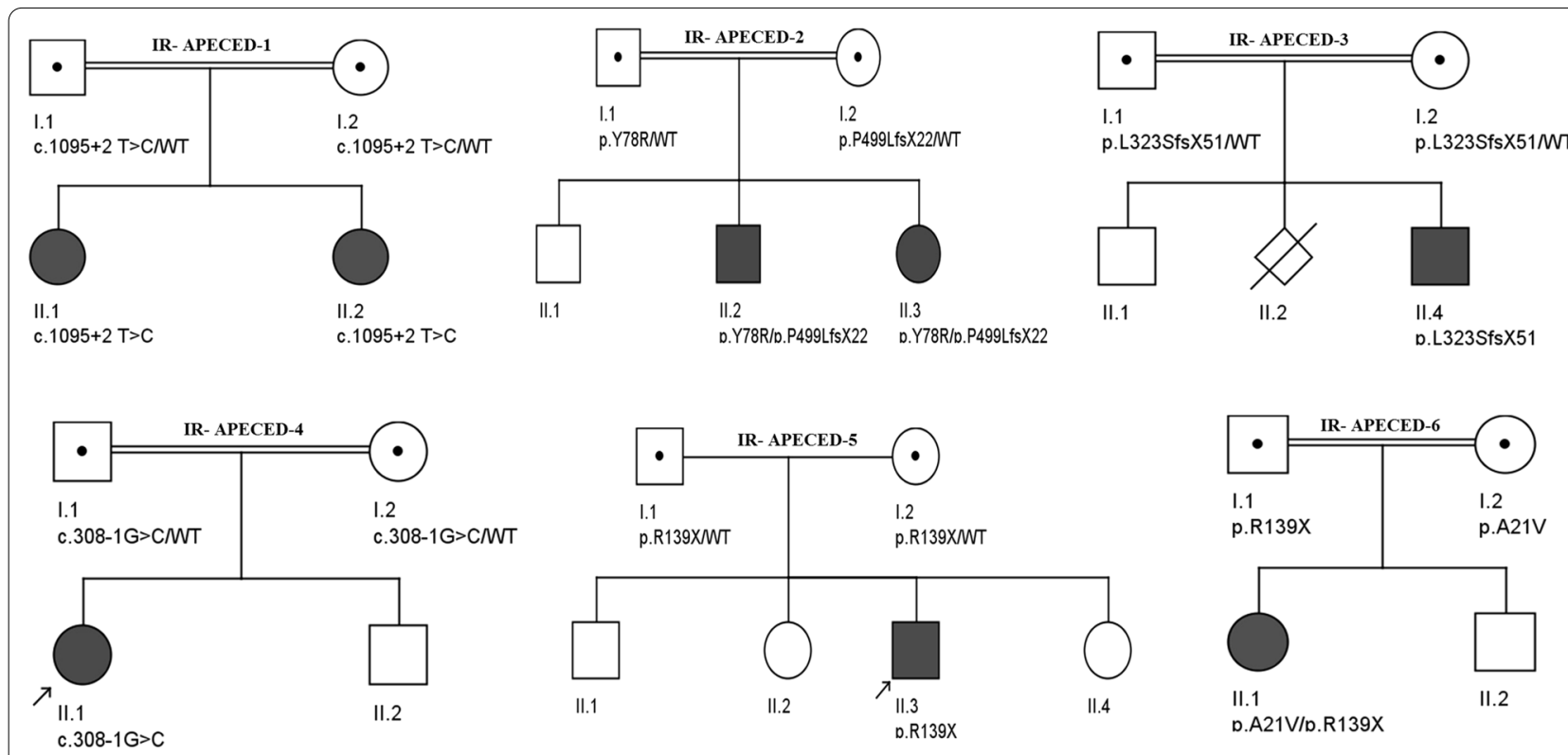

Fig. 1 Pedigrees of families APECED1-6. Circles—-female subjects; squares-male subjects; open circle and open square-normal; filled circle and filled square-affected 


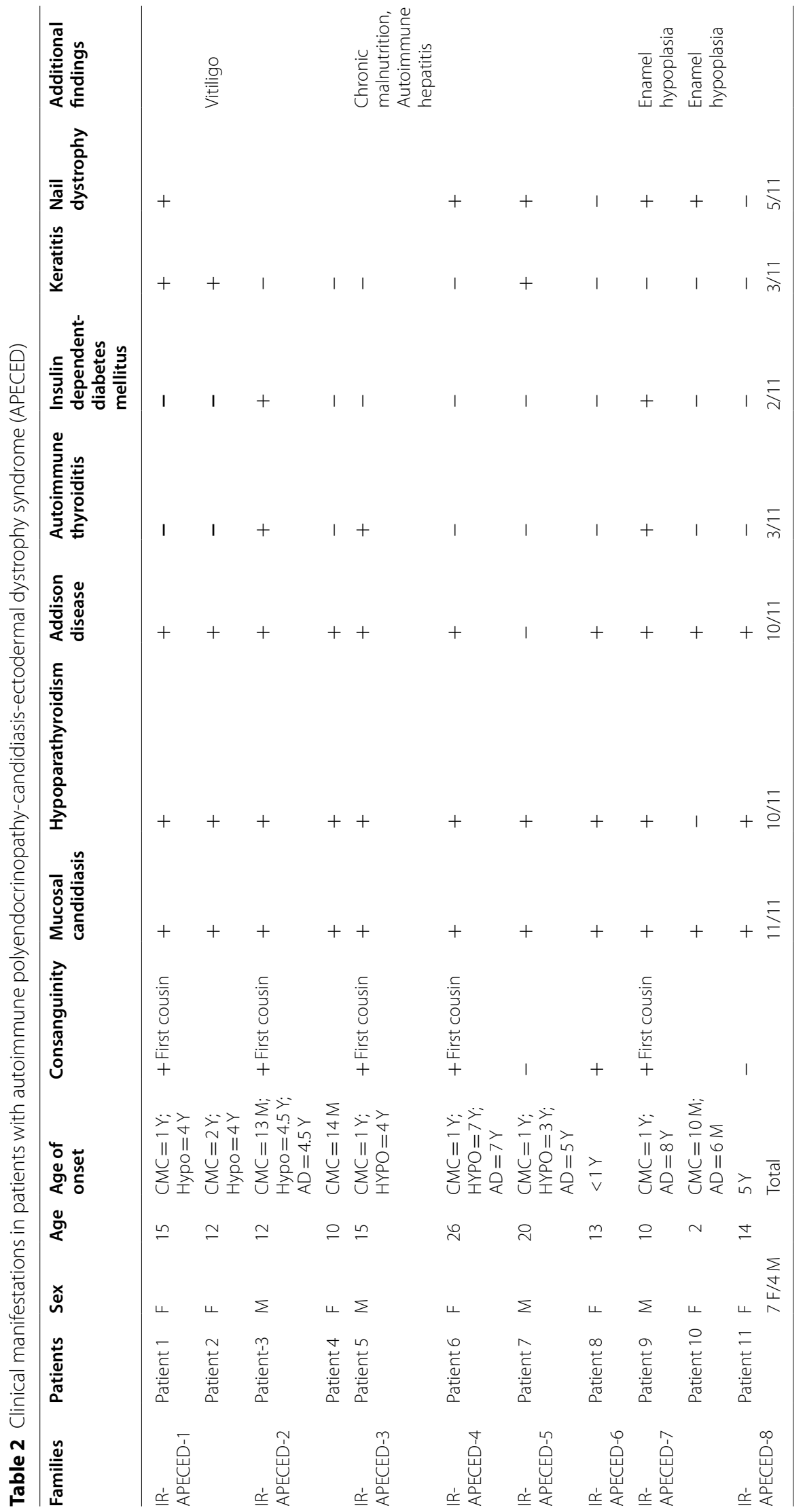


Table 3 Identified mutations in the AIRE gene from non-Jewish Iranian patients

\begin{tabular}{|c|c|c|c|c|c|c|}
\hline Families & Mutations Detected & Variant effect & Functional Consequence & Position & Genotype & Reference \\
\hline IR-APECED-1 & c. $1095+2 T>A$ & Splice donor loss & Splice donor variant & IVS9 & Hom & Seifi-Alan \\
\hline \multirow[t]{2}{*}{ IR-APECED-2 } & c.1496delC & p.P499LfsX22 & Stop gained & Exon 12 & Compound heterozygote & This study \\
\hline & c. $232 T>C$ & p.Y78R & Missense & Exon 2 & & Cihakova [12] \\
\hline IR-APECED-3 & $\begin{array}{l}\text { c.967_979delCTGTCCCCT } \\
\text { CCGC }\end{array}$ & p.L323SfsX51 & Frameshift variant & Exon 8 & Hom & Nagamine [5] \\
\hline IR-APECED-4 & $c .308-1 \mathrm{G}>C$ & Splice acceptor loss & Splice acceptor variant & IVS2 & Hom & This study \\
\hline IR-APECED-5 & c. $415 C>T$ & p.R139X & Stop gained & Exon 3 & Homo & Rosatelli [13] \\
\hline \multirow[t]{2}{*}{ IR-APECED-6 } & c. $415 C>T$ & p.R139X & Stop gained & Exon 3 & Compound heterozygote & Rosatelli [13] \\
\hline & c. $62 \mathrm{C}>\mathrm{T}$ & p.A21V & Missense & Exon 1 & & Halonen [30] \\
\hline
\end{tabular}

Novel variants identified in this study are shown in bold

critical role in regulating certain aspects of the immune system function. Therefore, mutations in the AIRE gene can reduce or eliminate function of the autoimmune regulator protein and will subsequently cause APECED [22]. Though mutations in AIRE are not common in many countries, this condition occurs more frequently in certain populations, affecting approximately 1 in 9000-25,000 individuals among Iranian Jews, Sardinians, and Finns.

By sequencing the complete coding region as well as the exon-intron boundaries of the AIRE gene, we found four homozygous and two compound heterozygote mutations in six out of eight pedigrees with APECED and the heterozygous forms were further confirmed in their parents. Except for c.1095+2T>A (intron 9), R139X (exon 3) and p.L323SfsX51 (exon 8) (pathogenic mutations in the AIRE gene that have been previously reported in the Iranian non-Jewish population [17, 23]), this study demonstrated, for the first time, other disease-causing mutations (Table 3) in non-Jewish Iranian families. This could be of particular importance for clinicians and patients with relevant phenotypes to consider the possibility of a wide range of APECED mutations in different ethnicities in regions of the Middle East such as Iran .

The variants we reported here were almost equally distributed among categories of missense (2 of 7), stopgain (2 of 7), splicing ( 2 of 7) and frameshift (1 of 7) variants. However, statistically analyzing the total number of variants extracted from ensemble.org demonstrated that missense variants are the most frequent variants within the AIRE gene, comprising $68 \%$ of variants, followed by splice site variants (Fig. 3).

Clinically, CMC, Addison's disease and hypoparathyroidism were the most frequent findings in our study population. These manifestations are also the most penetrant phenotypes reported in similar studies. On the other hand, here we demonstrated the complete triad in 10 out of 11 cases. This rate seems to be greater than the average percentage reported in the literature (up to 63\%). In accordance with our findings, Fardi Golyan et al. [23] reported a frequency of $80 \%$ for the classic triad in their Iranian APECED cases. Therefore, simultaneous presence of the three APECED cardinal signs can be regarded as a unique finding in the Iranian non-Jewish APECED patients. This finding needs to be further evaluated in larger sample sizes and populations.

In contrast to Iranian Jewish patients that rarely have mucocutaneous candidiasis, all of our non-Jewish patients, surprisingly, had mucocutaneous candidiasis. Researchers believe that differences in the effects of specific AIRE gene mutations, as well as variations in other genes that have not yet been identified, may help explain why the signs and symptoms of APECED can vary among affected individuals and populations. Thus, study of isolated populations, such as Iranian Jews, added to extended inbred pedigrees are useful aids for delineating genotype-phenotype correlations as well as mapping genes and genetic variations explaining the phenotypic heterogeneity of the disease [24]. However, findings in isolated populations might not be always valid in the general population [3]. In such cases, fine mapping in the general population, rather than focusing on genetically isolated populations, can be considered an alternative strategy for identification of genetic aberrations in rare monogenic disorders with Mendelian inheritance such as APECED.

In some cases, our results were in line with previous findings in other populations [3, 25]; nonetheless, occurrence of several new nucleotide variations as novel changes in the AIRE gene was also detected among our patients. For instance, results of recent studies in the Finnish population revealed that the $\mathrm{C}$ to $\mathrm{T}$ substitution at nucleotide 889 located in exon six is the most common mutation worldwide [26]. This mutation changes CGA into TGA as a stop codon and subsequently leads to a truncated 256 residue protein. This is found in about $85 \%$ of patients with APECED with a carrier frequency 
Table 4 Results of in silico analysis of the splice site variants found in this study

\begin{tabular}{llllll}
\hline Variant & Mutation taster & Human splice finder & BDGP & NetGen2 & CADD C-score $^{\text {a }}$ \\
\hline C.308-1G>C & Disease causing & Broken WT Acceptor Site & WT $=0.85 ;$ MUT $=$ NR & WT $=0.99 ;$ MUT = NR & 26.2 \\
C.1095+2T>A & Disease causing & Broken WT Donor Site & WT =0.97; MUT = NR & WT $=0.95 ;$ MUT = NR & 23.0 \\
\hline
\end{tabular}

${ }^{a}$ A score of greater or equal 20 indicates the $1 \%$ most deleterious and so on

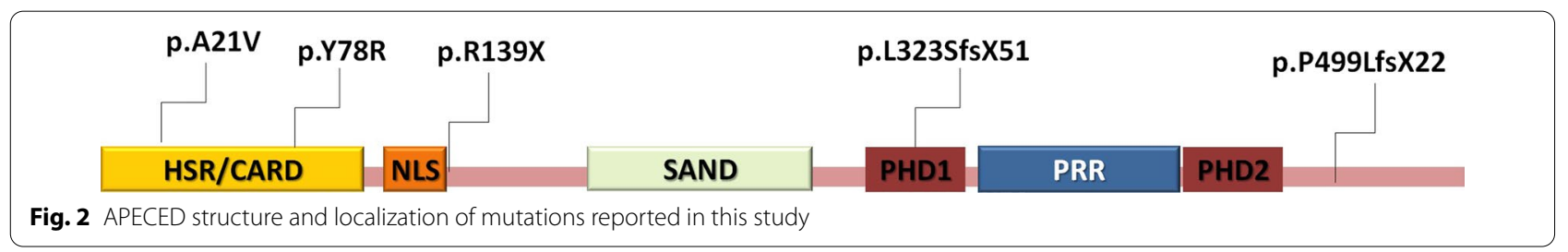

of 1:250 in randomly selected unaffected Finns [26]. Nonetheless, this mutation is not a rare polymorphism and, interestingly, it was not found in non-Jewish Iranian patients of this study.

A 13-bp deletion in exon eight has been found in a number of patients with APECED worldwide and is the second most common mutation in Finnish patients with APECED [27]. Based on results obtained in this study, this mutation has occurred in one out of eight nonJewish Iranian patients in homozygous form. This deletion occurs in the region encoding the first PHD finger (Fig. 2), resulting in a protein with no intact PHD finger [28].
Two out of the seven pathogenic variants observed here resided in splice sites. The novel pathogenic variant c.308-1G>C was located in the second intron of the AIRE gene. This splice acceptor variant represented a disease-causing score of 26.2 in the CADD algorithm. The $c .1095+2 \mathrm{~T}>\mathrm{A}$ substitution was a previously reported splice donor variant. This splice site change was predicted by in silico analysis to affect all downstream motifs in the protein including LXXLL and PHD domains. Also, by the CADD algorithm, this variation is predicted to be deleterious (score of 22.3). Interestingly, this variant was first identified in an Iranian APECED pedigree [17] and to the best of our knowledge has not been reported elsewhere so far. Thus, this variant can be subjected to

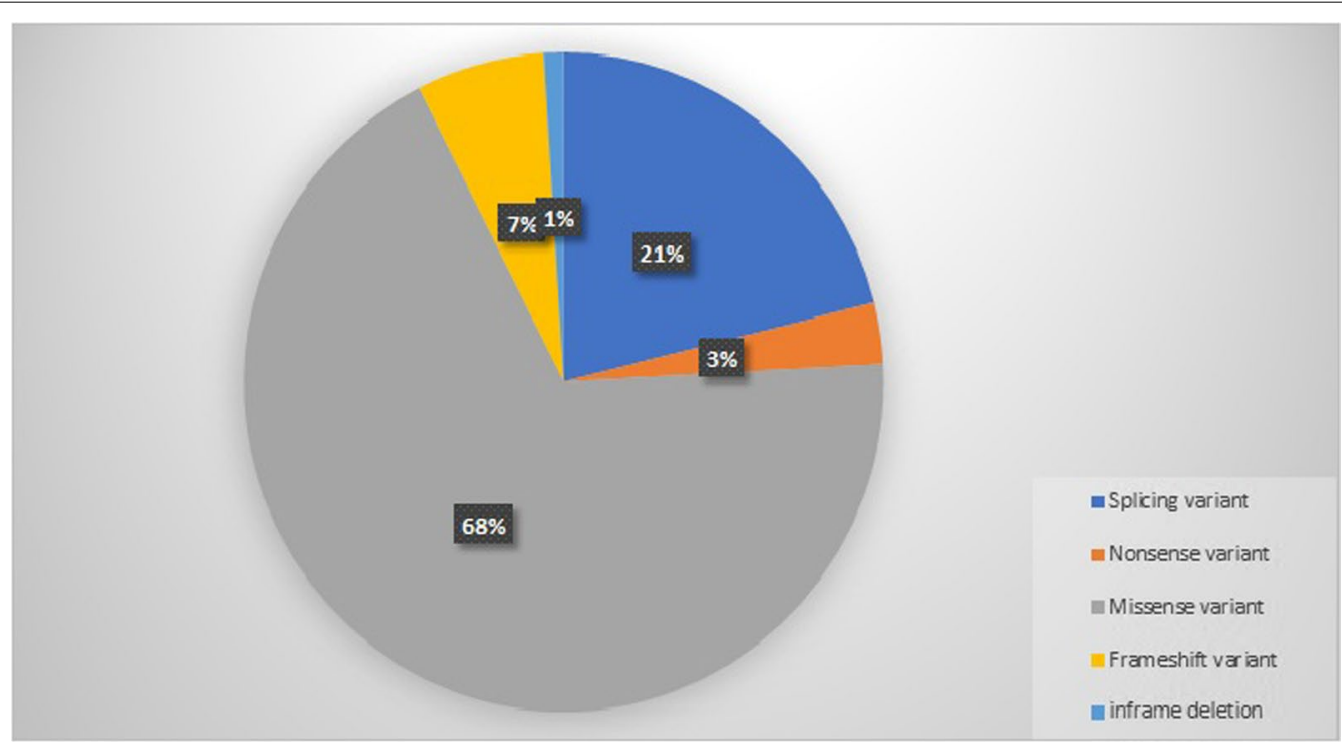

Fig. 3 Graph representing distribution of AIRE mutations based on mutation type 
further analysis as a putative founder mutation in the Iranian non-Jewish population.

Furthermore, in our patient series, we observed two homozygous missense mutations in the AIRE gene. These mutations are of particular interest because they may reveal functionally critical regions of the APECED protein. One of the mutations was c.232T $>C$ substitution in exon 2, identified in family No. 2, and has been previously reported to be Pathogenic/Likely Pathogenic in APECED 1. Substitution of c.62C $>\mathrm{T}$ in the affected child of family No. 6 was the other missense mutation in our study. These single nucleotide exchanges have diseasecausing prediction in both MutationTaster and CADD databases and are predicted to severely affect the HSR domain. This domain, is also present in the amino terminus of the human and mouse Sp100 and Sp140 proteins, is predicted to possess a function in regulation of gene expression and has recently been demonstrated to act as a homo-dimerization domain as well as being responsible for targeting these mentioned proteins to nuclear bodies. This dimerization might be crucial for DNA-binding or for binding to a currently unknown partner, and consequently, these mutations could disrupt such interactions. This clustering of missense mutations in the HSR domain provided further evidence for functional significance in the APECED protein $[29,30]$.

No evidence was found to prove any correlation between mutation type or mutation location with the clinical symptoms observed in our patients. Therefore, applying functional analysis of the APECED protein both in vitro and in animal models may elucidate mechanisms underlying molecular pathogenesis of this disease.

\section{Conclusion}

We identified two novel mutations and five other previously reported mutations (other ethnicities) in eight Iranian non-Jewish patients with APECED. No mutation was found in three other cases which may be suggestive of other loci being involved in pathogenesis of APECED in the Iranian non-Jewish population. Identification of new genetic variations in the coding region of the AIRE gene associated with APECED might facilitate genetic diagnosis, prognosis, and potential treatment of the disease. Moreover, these findings may further elucidate mechanisms underlying autoimmune disorders such as APECED.

\section{Acknowledgements}

The authors extend deep felt gratitude to the patients and their willingness to participate in this study.

\section{Authors' contributions}

A.S. contributed to patients'assessments and guided clinical data interpretation. SP performed the experiments, analyzed the data and drafted the manuscript. A.S. and S.P. have contributed equally to the work. F.V. contributed in molecular experiments and data analysis. N.J. performed in silico analysis and drafted the manuscript. F.B., A.S., F.S., F.A., P.R. and N.P. contributed to patients' assessments. H.A. and M.A. contributed to molecular experimental design and analyzed the data. M.T. designed and supervised the study, drafted and finalized the manuscript. All authors read and approved the final manuscript.

\section{Funding}

This project did not receive any financial support and was self-funded.

\section{Availability of data and materials}

Supporting information are available from the corresponding author upon request.

\section{Declarations}

Ethics approval and consent to participate

The study protocol was approved by the ethics committee of Babol University of Medical Sciences. The study consent forms were attained from the participants and all participants signed informed consent forms.

\section{Consent for publication}

Consent to publish has been obtained from participants to report individual patient data.

\section{Competing interests}

The authors declare that they have no competing interests.

\section{Author details}

${ }^{1}$ Division of Endocrinology and Metabolism, Growth and Development Research Center, Children's Medical Center, Tehran University of Medical Sciences, Tehran, Iran. ${ }^{2}$ Cellular and Molecular Biology Research Center, Health Research Institute, Babol University of Medical Sciences, Babol, Iran. ${ }^{3}$ Department of Medical Genetics, School of Medicine, Tehran University of Medical Sciences, Tehran, Iran. ${ }^{4}$ Department of Clinical Biochemistry, School of Medicine, Kermanshah University of Medical Sciences, Kermanshah, Iran.

${ }^{5}$ Growth and Development Research Center, Children's Medical Center, Tehran University of Medical Sciences, Tehran, Iran. ${ }^{6}$ Division of Allergy and Clinical Immunology, Department of Pediatrics, Children's Medical Center, Tehran University of Medical Sciences, Tehran, Iran. ${ }^{7}$ Department of Medical Genetics, Faculty of Medicine, Babol University of Medical Sciences, Babol, Iran.

Received: 11 June 2021 Accepted: 19 December 2021

Published online: 06 January 2022

References

1. Arstila TP, Jarva H. Human APECED; a sick thymus syndrome? Front Immunol. 2020;4:66.

2. Guo C-J, Leung PS, Zhang W, Ma X, Gershwin ME. The Immunobiology and clinical features of type 1 autoimmune polyglandular syndrome (APS-1). Autoimmun Rev. 2020;6:66.

3. Björses $\mathrm{P}$, Halonen M, Palvimo JJ, Kolmer M, Aaltonen J, Ellonen P, et al. Mutations in the AIRE gene: effects on subcellular location and transactivation function of the autoimmune polyendocrinopathy-candidiasisectodermal dystrophy protein. Am J Hum Genet. 2000;66(2):378-92.

4. Zlotogora J, Shapiro MS. Polyglandular autoimmune syndrome type I among Iranian Jews. J Med Genet. 1992;29(11):824-6.

5. Nagamine K, Pr Peterson, Scott HS, Kudoh J, Minoshima S, Heino M, et al. Positional cloning of the APECED gene. Nat Genet. 1997;17(4):393-8.

6. Pr Peterson, Nagamine K, Scott H, Heino M, Kudoh J, Shimizu N, et al. APECED: a monogenic autoimmune disease providing new clues to selftolerance. Immunol Today. 1998;19(9):384-6.

7. Peterson P, Pitkänen J, Sillanpää N, Krohn KJC. Autoimmune polyendocrinopathy candidiasis ectodermal dystrophy (APECED): a model disease to study molecular aspects of endocrine autoimmunity. Clin Exp Immunol. 2004;135(3):348. 
8. Moraes-Vasconcelos D, Costa-Carvalho B, Torgerson T, Ochs H. Primary immune deficiency disorders presenting as autoimmune diseases: IPEX and APECED. J Clin Immunol. 2008;28(1):11-9.

9. Oven I, Brdičková N, Kohoutek J, Vaupotič T, Narat M, Peterlin BMJM, et al. AIRE recruits P-TEFb for transcriptional elongation of target genes in medullary thymic epithelial cells. Mol Cell Biol. 2007;27(24):8815-23.

10. Heino M, Pr Peterson, Kudoh J, Shimizu N, Antonarakis SE, Scott HS, et al. APECED mutations in the autoimmune regulator (AIRE) gene. Hum Mutat. 2001;18(3):205-11.

11. Zaidi G, Sahu R, Zhang L, George G, Bhavani N, Shah N, et al. Two novel AIRE mutations in autoimmune polyendocrinopathy-candidiasis-ectodermal dystrophy (APECED) among Indians. Clin Genet. 2009;76(5):441-8.

12. Cihakova D, Trebusak K, Heino M, Fadeyev V, Tiulpakov A, Battelino T, et al. Novel AIRE mutations and P450 cytochrome autoantibodies in Central and Eastern European patients with APECED. Hum Mutat. 2001;18(3):225-32.

13. Rosatelli MC, Meloni A, Meloni A, Devoto M, Cao A, Scott HS, et al. A common mutation in Sardinian autoimmune polyendocrinopathy-candidiasis-ectodermal dystrophy patients. Hum Genet. 1998;103(4):428-34.

14. Meyer G, Donner H, Herwig J, Böhles H, Usadel K, Badenhoop KJC. Screening for an AIRE-1 mutation in patients with Addison's disease, type 1 diabetes, Graves' disease and Hashimoto's thyroiditis as well as in APECED syndrome. Clin Endocrinol. 2001;54(3):335-8.

15. Husebye ES, Perheentupa J, Rautemaa R, et al. Clinical manifestations and management of patients with autoimmune polyendocrine syndrome type I. J Intern Med. 2009;265(5):514-29.

16. Björses P, Aaltonen J, Horelli-Kuitunen N, Yaspo M-L, Peltonen LJ. Gene defect behind APECED: a new clue to autoimmunity. J Intern Med. 1998;7(10):1547-53

17. Seifi-Alan M, Shamsi R, Setoodeh A, Sayarifard F, Aghasi P, Kompani F, et al. Autoimmune polyendocrinopathy-candidiasis-ectodermal dystrophy: report of three cases from Iran. J Intern Med. 2016;29(8):979-83.

18. Ahonen P, Myllärniemi S, Sipilä I, Perheentupa JJ. Clinical variation of autoimmune polyendocrinopathy-candidiasis-ectodermal dystrophy (APECED) in a series of 68 patients. N Engl J Med. 1990;322(26):1829-36.

19. Miller SA, Dykes DD, Polesky HF. A simple salting out procedure for extracting DNA from human nucleated cells. Nucleic Acids Res. 1988;16(3):1215.

20. Richards S, Aziz N, Bale S, Bick D, Das S, Gastier-Foster J, et al. Standards and guidelines for the interpretation of sequence variants: a joint consensus recommendation of the American College of Medical Genetics and Genomics and the Association for Molecular Pathology. Genet Med. 2015;17(5):405-24.

21. Kisand K, Peterson PJ. Autoimmune polyendocrinopathy candidiasis ectodermal dystrophy. J Clin Immunol. 2015;35(5):463-78.

22. Pellegrino M, Bellacchio E, Dhamo R, Frasca F, Betterle C, Fierabracci AJ. A novel homozygous mutation of the AIRE gene in an apeCeD patient from Pakistan: case report and review of the literature. J Clin Immunol. 2018:9:1835.

23. Fardi Golyan F, Ghaemi N, Abbaszadegan MR, Dehghan Manshadi SH, Vakili R, Druley TE, et al. Novel mutation in AIRE gene with autoimmune polyendocrine syndrome type 1. Immunobiology. 2019;224(6):728-33.

24. Eaves IA, Merriman TR, Barber RA, Nutland S, Tuomilehto-Wolf E, Tuomilehto J, et al. The genetically isolated populations of Finland and Sardinia may not be a panacea for linkage disequilibrium mapping of common disease genes. Nat Genet. 2000;25(3):320-3.

25. Krohn K, Heino M, Peterson P, Scott HS, Antonarakis SE, Lalioti MD, et al. Gene defective in APECED and its use. Google Patents; 2010.

26. Krohn K, Heino M, Peterson Pr, Scott HS, Antonarakis SE, Lalioti MD, et al. Gene defective in APECED and its use. Google Patents.

27. Pearce SH, Cheetham T, Imrie H, Vaidya B, Barnes ND, Bilous RW, et al. A common and recurrent 13-bp deletion in the autoimmune regulator gene in British kindreds with autoimmune polyendocrinopathy type 1. Am J Hum Genet. 1998;63(6):1675-84.

28. Li D, Streeten EA, Chan A, Lwin W, Tian L, Pellegrino da Silva R, Kim CE, Anderson MS, Hakonarson $\mathrm{H}$, Levine MA. Exome sequencing reveals mutations in AIRE as a cause of isolated hypoparathyroidism. J Clin Endocrinol Metab. 2017;102(5):1726-33.

29. Ilmarinen T. Functional and cellular analysis of autoimmune regulator (AIRE) protein. 2007
30. Halonen $\mathrm{M}$, Kangas $\mathrm{H}$, Rüppell $\mathrm{T}$, Ilmarinen $\mathrm{T}$, Ollila J, Kolmer M, et al. APECED-causing mutations in AIRE reveal the functional domains of the protein. Hum Mutat. 2004:23(3):245-57.

\section{Publisher's Note}

Springer Nature remains neutral with regard to jurisdictional claims in published maps and institutional affiliations.
Ready to submit your research? Choose BMC and benefit from:

- fast, convenient online submission

- thorough peer review by experienced researchers in your field

- rapid publication on acceptance

- support for research data, including large and complex data types

- gold Open Access which fosters wider collaboration and increased citations

- maximum visibility for your research: over $100 \mathrm{M}$ website views per year

At BMC, research is always in progress.

Learn more biomedcentral.com/submissions 